\title{
CD44 alternative splicing and hnRNP A1 expression are associated with the metastasis of breast cancer
}

\author{
TIING JEN LOH ${ }^{1}$, HEEGYUM MOON ${ }^{1}$, SUNGHEE CHO ${ }^{1}$, HANA JANG $^{1}$, YONG CHAO LIU ${ }^{1}$, \\ HONGMEI TAI ${ }^{2}$, DA-WOON JUNG ${ }^{1}$, DARREN R. WILLIAMS ${ }^{1}$, HEY-RAN KIM ${ }^{3}$, MYUNG-GEUN SHIN ${ }^{3}$, \\ D. JOSHUA LIAO ${ }^{4}$, JIANHUA ZHOU ${ }^{5}$, WEI SHI ${ }^{6}$, XUEXIU ZHENG ${ }^{1}$ and HAIHONG SHEN ${ }^{1}$ \\ ${ }^{1}$ School of Life Sciences, Gwangju Institute of Science and Technology, Gwangju, Republic of Korea; ${ }^{2}$ Department of \\ Endocrinology, Yanji Hospital, Jilin, P.R. China; ${ }^{3}$ Department of Laboratory Medicine, Chonnam National University \\ Medical School and Chonnam National University Hwasun Hospital, Hwasun, Republic of Korea; ${ }^{4}$ Hormel Institute, \\ University of Minnesota, Austin, MN, USA; ${ }^{5}$ Jiangsu Key Laboratory of Neuroregeneration, Nantong University, \\ Nantong, Jiangsu; ${ }^{6}$ Key Laboratory for Molecular Enzymology and Engineering, The Ministry of Education, \\ College of Life Sciences, Jilin University, Changchun, Jilin, P.R. China
}

Received March 19, 2015; Accepted May 20, 2015

DOI: $10.3892 / o r .2015 .4110$

\begin{abstract}
CD44 is a transmembrane receptor for hyaluronic acid. CD44 pre-mRNA contains 19 exons, 9 of which are alternatively spliced. Among the CD44 spliced variants, the v4-7 variant, one of the $\mathrm{v} 6$ exon-containing isoforms that contains variable exon 4, 5, 6 and 7, confers metastatic potential to nonmetastatic cells. Splicing of CD44 and the function of CD44 isoforms are different in breast cancer cells. hnRNP A1 is a ubiquitously expressed protein with an inhibitory function in pre-mRNA splicing. We showed that CD44v6 isoform, which includes all of the v6-containing mRNA isoforms, had the highest expression level in non-metatatic breast cancer cells (MCF7) when compared to the level in metastatic breast cancer cells (MDA-MB-231) and normal breast cells (MCF10A). Furthermore we showed that hnRNP A1 knockdown regulated splicing of CD44 differently in breast cancer cells. We showed here that CD44 isoform expression is completely different in MDA-MB-231 cells than that in MCF7 and MCF10A cells, whereas MCF7 and MCF10A cells had a similar expression pattern of CD44 isoforms. RT-PCR analysis of CD44v6 showed that MCF7 and MCF10A cells predominantly expressed the c5v6v7v8v9v10c6 isoform. However, in addition to this isoform, MDA-MB-231 cells also expressed the c5v6v8v9v10c6 and c5v6c6 isoforms. We also found that knockdown of hnRNP A1 significantly reduced the expression
\end{abstract}

Correspondence to: Professor Haihong Shen or Dr Xuexiu Zheng, School of Life Sciences, Gwangju Institute of Science and Technology, Gwangju 500-712, Republic of Korea

E-mail: haihongshen@gist.ac.kr

E-mail: xuexiuzheng@gist.ac.kr

Key words: hnRNP A1, CD44, breast cancer of $\mathrm{c} 5 \mathrm{v} 6 \mathrm{v} 7 \mathrm{v} 8 \mathrm{v} 9 \mathrm{v} 10 \mathrm{c} 6$ and $\mathrm{c} 5 \mathrm{v} 6 \mathrm{v} 8 \mathrm{v} 9 \mathrm{v} 10 \mathrm{c} 6$, and promoted the expression of c5v6c6. hnRNP A1 knockdown significantly induced cell death. In addition, hnRNP A1 knockdown induced a decrease in cell invasion in the MDA-MB-231 cells. Our results indicate that the knockdown of hnRNP A1 has a specific function on the splicing of CD44 in breast cancer cells.

\section{Introduction}

Alternative splicing is a process during which exons are included in different mRNA isoforms to produce various proteins from a single gene $(1,2)$. Alternative splicing contributes to protein diversity. In alternative splicing, different $5^{\prime}$ splice-sites or 3' splice-sites are selected to induce exon inclusion or skipping $(3,4)$. Inclusion/skipping of exons are regulated by cis-acting or trans-acting elements $(5,6)$. Cis-acting elements include the RNA sequences on pre-mRNA that enhance or reduce exon inclusion/skipping. Exon splicing enhancer (ESE), exon splicing inhibitor (ESI), intron splicing enhancer (ISE) and intron splicing inhibitor (ISI) are the cis-acting elements. Trans-acting elements are the proteins that enhance or reduce alternative splicing. Arginine-serine-rich (SR) proteins and heterogeneous nuclear ribonucleoproteins (hnRNPs) are the best known trans-acting elements (7). These proteins strengthen or inhibit splice site recognition by the spliceosome (8). The variance of the sequences that define the exons and introns, the selection of the splice sites are regulated by the trans-acting elements. Alternative splicing is involved in a variety of biological processes including the cell cycle, energy transport, development and signal transduction. Misregulation of alternative splicing causes human diseases including cancer, especially breast cancer (9-11). Alternative splicing is engaged in specific cellular programs, including apoptosis, cell growth, angiogenesis, cell motility and EMT. It has been shown that alternative splicing of the survivin gene that produces variants with pro- and anti-apoptotic properties 
is altered in breast cancer and makes tumor cells resistant or sensitive to apoptosis. The splicing of the CD44 gene is altered in breast cancer and thus can be used as a prognostic marker.

CD44 is a member of a large family of cell adhesion molecules that mediate the communication and adhesion between adjacent cells and between cells and the extracellular matrix. CD44 also directs intracellular signaling for growth and motility. Thus, CD44 is involved in many types of cancers including breast cancer. CD44 pre-mRNA encodes a group of proteins ranging from 80 to $200 \mathrm{kDa}$ in size (12-14). The heterogeneity of this group is produced by alternative splicing. CD44 pre-mRNA includes 20 exons, which encode a huge number of CD44 isoforms (15). Exons 1-5 (c1-c5) and 16-20 (c6-c10) are constant exons, whereas exons 6-15 (v1-v10) are variant exons (16). CD44v creates larger isoforms and exposing-binding sites for additional post-translational modifications and ligand-binding sites. Variant expression is regulated by tissue and environment-specific factors and oncogenic pathways such as the Ras-MAPK cascade (17-19). CD44 mediates signaling through the oncogene c-Met (20). Among the CD44 variants, splicing of CD44v6 that includes all of the v6-containing mRNA isoforms, plays an important role. For example, Met, the receptor for hepatocyte growth factor (HGF), is overexpressed in $20-30 \%$ of breast cancers, is associated with poor clinical outcome, and requires CD44v6 to become fully activated. CD44v6-specific antibodies block Met activation in many different cancer cell lines (21). CD44v6 has been shown to activate endothelial cell migration, sprouting and tubule formation through activation of c-Met and VEGFR-2 in response to HGF or VEGF-A (22). The function of CD44v6 in breast cancer is different from other cancers. For example, transfection of CD44 variants into a non-metastatic rat pancreatic carcinoma cell line was found to confer metastatic potential to these cells when infected into syngeneic rats (23). The metastasis was blocked by treatment with the anti-v6 monoclonal antibody (24). However, research on breast cancer has conflicting results (25). Overexpression of CD44s was induced in the non-metastatic MCF7 breast cancer cell line, and the induction of CD44s, in addition to promoting aggressive characteristics in vitro (26), promoted metastasis to the liver when injected into immune-deficient mice, although it did not affect the growth rate or local invasion (27).

hnRNP A1 is a member of the hnRNP family. hnRNP A1 inhibits splicing through binding to the target RNA (28). hnRNP A1 represses splicing by promoting distal splice site selection, blocking the assembly of the splicing complex through the recruitment of snRNPs, and looping out entire exons $(29,30)$. hnRNP A1 promotes tumor invasion through regulation of CD44v6 and is an indicator of poor prognosis in hepatocellular carcinoma (31).

Since the function of the CD44 variants in breast cancer cells is different from other types of cancers, we studied the function of hnRNP A1 in breast cancers. Here, we showed that CD44 isoform expression was completely different in metastatic breast cancer cells (MDA-MB-231) when compared with that in non-metastatic breast cancer (MCF7) and normal breast cells (MCF10A), whereas MCF7 and MCF10A cells had a similar expression pattern for CD44 isoforms. We found that knockdown of hnRNP A1 significantly reduced the expres-
Table I. Oligonucleotides used for cDNA synthesis and PCR reactions.

\begin{tabular}{ll}
\hline Primer name & \multicolumn{1}{c}{ Oligonucleotide sequences $\left(5^{\prime} \rightarrow 3^{\prime}\right)$} \\
\hline CD44 RT & ATG CAA ACT GCA AGA ATC \\
STD CD44c5 For & AAGACATCTACCCCAGCAAC \\
STD CD44c7 Rev & TTTGCTCCACCTTCTTGACTCC \\
v2 & GAT GAG CAC TAG TGC TAC AG \\
v3 & ACG TCT TCA AAT ACC ATC TC \\
v4 & TCA ACC ACA CCA CGG GCT TT \\
v5 & GTA GAC AGA AAT GGC ACC AC \\
v6 & CAG GCA ACT CCT AGT AGT AC \\
v7 & CAG CCT CAG CTC ATA CCA G \\
v8 & TCC AGT CAT AGT ACA ACG CT \\
v9 & CAG AGC TTC TCT ACA TCA CA \\
v10 & GGT GGA AGA AGA GAC CCA AA \\
CD44c5v6 For & ATCCCTGCTACCATCCAGGCAAC \\
GAPDH For & ACC ACA GTC CAT GCC ATC A \\
GAPDH Rev & TCC ACC ACC CTG TTG CTG TA \\
hnRNP A1 For & ACA TAT GCC ACT GTG GAG GAG \\
hnRNP A1 Rev & CTT GCT TTG ACA GGG CTT TTC
\end{tabular}

For, forward; Rev, reverse; hnRNP, heterogeneous nuclear ribonucleoprotein.

sion of c5v6v7v8v9v10c6 and c5v6v8v9v10c6, and promoted expression of c5v6c6. hnRNP A1 knockdown induced significant cell death and a decrease in the invasion of MDA-MB-231 cells. Our results indicate that the function of hnRNP A1 is unique in breast cancer cells.

\section{Materials and methods}

Cell culture. MCF10A cells were maintained in DMEM/F12 medium supplemented with $5 \%$ horse serum, $20 \mathrm{ng} / \mathrm{ml} \mathrm{EGF}$, $0.5 \mathrm{mg} / \mathrm{ml}$ hydrocortisone, $100 \mathrm{ng} / \mathrm{ml}$ cholera toxin and $10 \mu \mathrm{g} / \mathrm{ml}$ insulin. MCF7 and 293T cells were grown in Dulbecco's modified Eagle's medium (DMEM) supplemented with $10 \%$ fetal bovine serum (FBS). MDA-MB-231 cells were grown in RPMI-1640 medium supplemented with $10 \% \mathrm{FBS}$ at $37^{\circ} \mathrm{C}$ in a humidified $5 \% \mathrm{CO}_{2}$ condition.

$R T-P C R$. Total RNA was extracted using RiboEx reagent (GeneAll) following the manufacturer's instructions. One microgram of total RNA was reverse transcribed using oligodT primer using ImProm-II ${ }^{\mathrm{TM}}$ reverse transcriptase (Promega) following the manufacturer's instructions. One microliter of cDNA was amplified by PCR using G-Taq polymerase (Cosmo Genetech). The primers used are listed in Table I. For endogenous CD44, RT-PCR was conducted as previously described (32). Briefly, $1 \mu \mathrm{g}$ of total RNA was reverse transcribed using the CD44RT primer and AMV reverse transcriptase (Promega) according to the manufacturer's instructions. One microliter of cDNA was amplified by PCR using G-Tag. The endogenous RT-PCR products were confirmed by sequencing. 
A
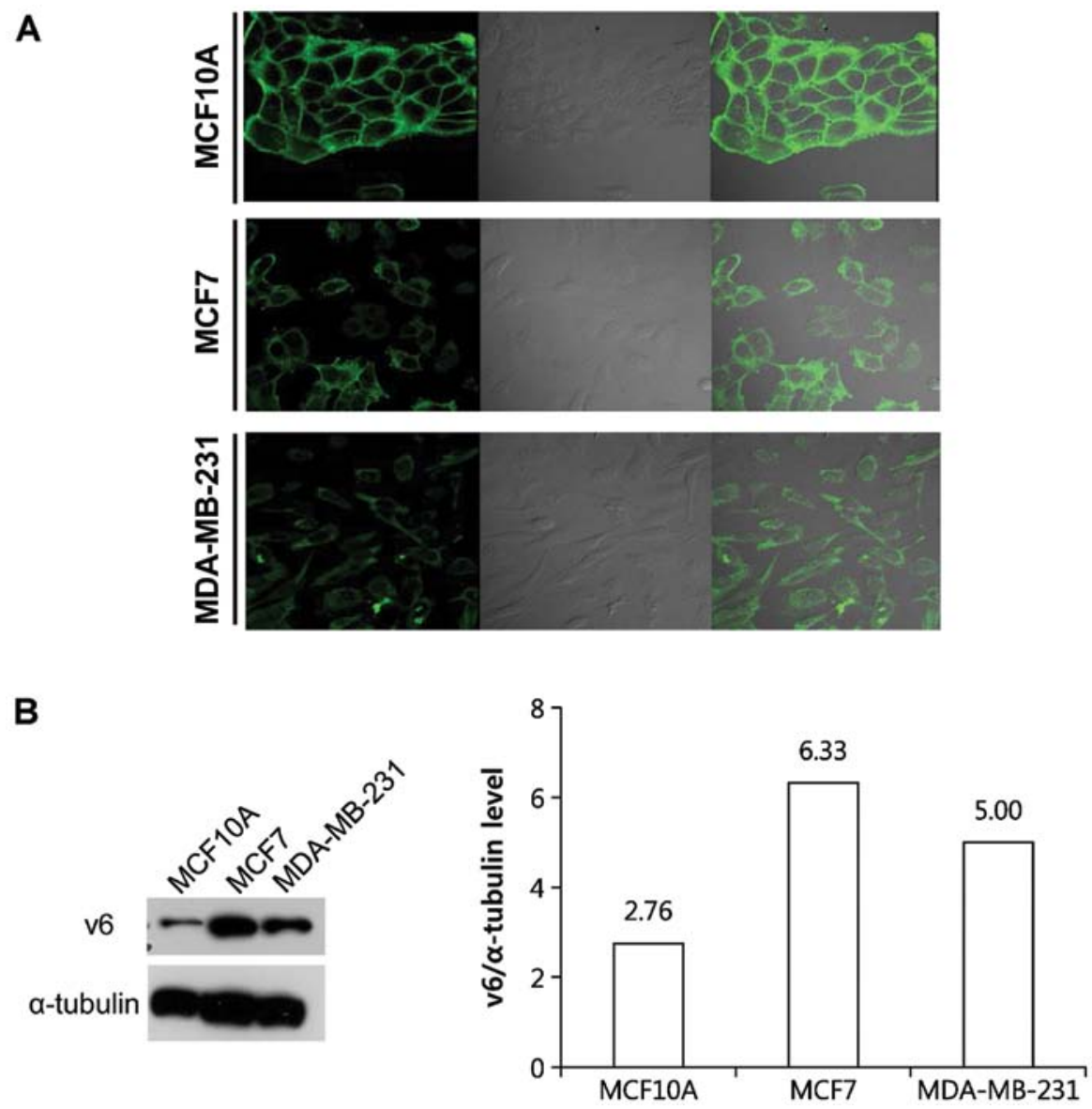

Figure 1. CD44v6 is expressed most abundantly in non-metastatic breast cancer cells (MCF7). (A) Immunohistochemistry using an anti-CD44-v6 antibody in MCF10A, MCF7 and MDA-MB-231 cells. (B) Immunoblotting with an anti-CD44-v6 antibody in MCF10A, MCF7 and MDA-MB-231 cells. Quantitation of the results is shown to the right.

shRNA treatment. The shRNA lentiviruses were generated by co-transfection of the pLKO.1 plasmid that encodes the hnRNP A1 mRNA matching or non-silencing sequence (Open Biosystems). PSPAX2 and PMD2G plasmids were transfected into 293T cells using Effectene reagent (Qiagen) according to the manufacturer's instructions. The media were changed after $24 \mathrm{~h}$ and incubation was carried out for another $24 \mathrm{~h}$. The supernatants containing the lentiviruses were harvested using a $0.45-\mu \mathrm{m}$ filter. The cells were seeded in a 6 -well plate one day before infection. The lentiviral-containing supernatants were added to the cells supplemented with $8 \mu \mathrm{g} / \mathrm{ml}$ Polybrene. After $72 \mathrm{~h}$ of infection, the RNAs were extracted for RT-PCR or further experiments.

Invasion and migration assays. The Transwell invasion and migration assays were conducted on MDA-MB-231 cells following siRNA treatment. In the invasion analysis, Transwell chambers with an $8-\mu \mathrm{m}$ pore size were coated with $50 \mu \mathrm{g} / \mathrm{ml}$ collagen type I. In the migration assay, Transwell chambers were coated with $50 \mu \mathrm{g} / \mathrm{ml}$ fibronectin. Briefly, DMEM containing $10 \%$ FBS was added to the lower well chambers for inducing cancer cell invasion. Cells in DMEM without FBS were added to the upper chambers. After incubation for $48 \mathrm{~h}$ in the invasion assay or $24 \mathrm{~h}$ in the migration assay, the migrated cells in the lower chamber were fixed with $3.7 \%$ formaldehyde for $1 \mathrm{~h}$ prior to staining with $0.01 \%$ crystal violet for $1 \mathrm{~h}$.
Cell proliferation and viability assay. Methylthiazolyldiphenyl-tetrazolium bromide (MTT) $(50 \mu \mathrm{g} / \mathrm{ml}$; Sigma-Aldrich) solution was added to the cell growth media and incubated for $3 \mathrm{~h}$ at $37^{\circ} \mathrm{C}$ until purple precipitate was visible. After removing the MTT reagent, $200 \mu 1$ dimethyl sulfoxide (DMSO) was added to dissolve the purple precipitate. Absorbance at $570 \mathrm{~nm}$ was recorded.

Immunoblotting. MCF10A, MCF7 and MDA-MB-231 cells were lysed with ice-cold lysis buffer $(0.1 \%$ Triton $\mathrm{X}-100$, $50 \mathrm{mM}$ Tris- $\mathrm{Cl} \mathrm{pH} 7.5,150 \mathrm{mM} \mathrm{NaCl}, 5 \mathrm{mM}$ EDTA and $1 \mathrm{mM} \beta$-mercaptoethanol) for $20 \mathrm{~min}$ at $4^{\circ} \mathrm{C}$ and then centrifuged at $12,000 \mathrm{rpm}$ for $30 \mathrm{~min}$ at $4^{\circ} \mathrm{C}$. The supernatants were mixed with SDS sample buffer and separated on $12 \%$ SDS-PAGE gels. The gel was transferred to a pre-activated PVDF membrane. The membranes were blocked using 5\% skim milk in TBST solution and then incubated with the anti-CD44v6 (VFF-18; eBioscience) or the anti-hnRNP A1 (sc-32301; Santa Cruz Biotechnology) antibody in TBST for $2 \mathrm{~h}$. After washing, the membranes were incubated with horseradish peroxidase-conjugated secondary antibody for $1 \mathrm{~h}$. The specific proteins were visualized by ECL western blotting detection reagents after exposure to X-ray film.

Immunofluorescence. Coverslips were pre-incubated in a 24-well plate containing DMEM overnight. MCF10A, MCF7 


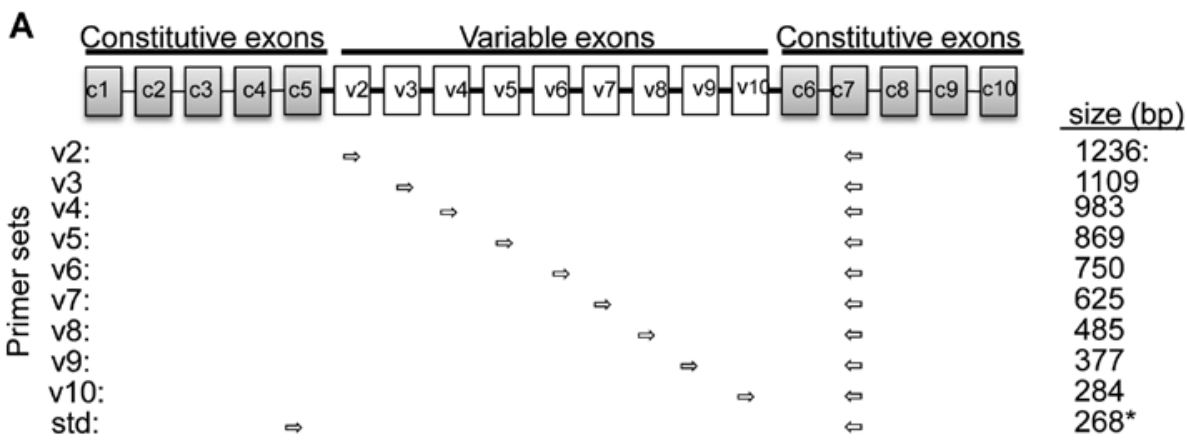

B

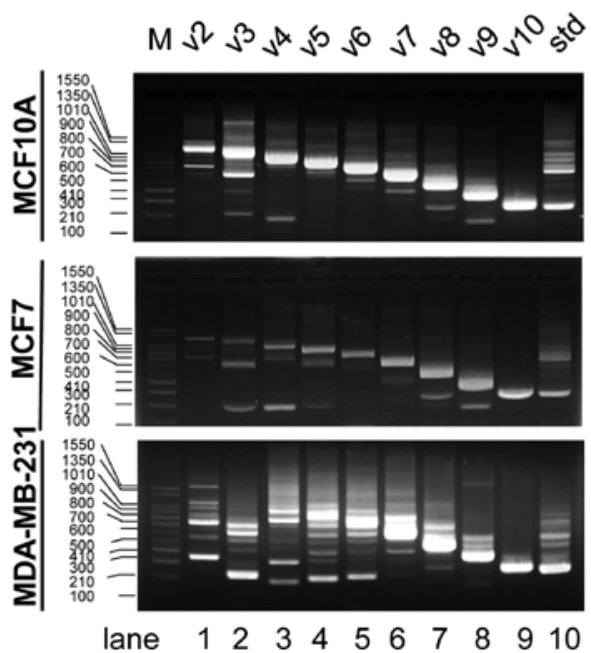

Figure 2. MDA-MB-231 cells show a differential expression profile of CD44 isoforms when compared with that of the MCF7 and MCF10A cells. (A) Primer positions are shown. The expected sizes of the PCR products are indicated. (B) RT-PCR results of the CD44v isoforms using the different primers as shown in $\mathrm{A}$ in the MCF10A, MCF7 and MDA-MB-231 cells.

and MDA-MB-231 cells were seeded into the plates and incubated overnight after removing the media. The cells were fixed with $4 \%$ paraformaldehyde (PFA) for $20 \mathrm{~min}$ after washing with PBS. Blocking was performed with $2 \%$ BSA at room temperature by shaking for $1 \mathrm{~h}$. Anti-CD44-v6 antibody in $1 \%$ BSA was added to each well and incubated overnight at $4^{\circ} \mathrm{C}$. The anti-mouse-lgG-FITC (Santa Cruz Biotechnology) in 1\% BSA was then added and incubated for $50 \mathrm{~min}$. The coverslips were then fixed on the slide with mounting media and dried in the dark. Images were examined using a confocal microscope (Olympus FV-1000) with 60x lens.

\section{Results}

CD44v6 is expressed most abundantly in non-metastatic breast cancer cells (MCF7). In order to identify the function of CD44-v6 protein, which includes all of the v6 exon containing CD44 proteins, in the progression and metastasis of breast cancer, we compared CD44-v6 expression in normal breast (MCF10A), non-metastatic breast cancer (MCF7) and metastatic breast cancer cells (MDA-MB-231). In the first set of experiments, we compared the expression of CD44v6 in these cells. To identify the location of the CD44v6 isoforms, we performed immunohistochemistry with an anti-CD44-v6 antibody. Fig. 1A shows that CD44v6 was highly expressed on the cell membrane in the MCF10A cells as compared with that in the MCF7 and MDA-MB-231 cells, in which CD44v6 was mostly expressed in the cytoplasm. In the second set of experiments, we performed immunoblot analysis with an anti-CD44-v6 antibody of the cell lysates from these three cell lines. As shown in Fig. 1B, MCF10A cells expressed the lowest level of CD44-v6 protein (2.76\% of v6/tubulin). MCF7 cells showed the highest expression level of CD44-v6 (6.33\% of v6/tubulin), whereas MDA-MB-231 cells expressed a moderate level of CD44-v6 (5\% of v6/tubulin). We concluded that the expression level of CD44-v6 protein was not linked to the metastatic potential of human breast cancer cells.

MDA-MB-231 cells exhibit differential expression of CD44v6 isoforms when compared to that of either the MCF7 or MCF10A cells. We next compared the expression of different CD44v6 isoforms, which include all v6 exon-containing isoforms, in these three cell lines. We designed serials of forward primers which base pair with v2, v3, v4, v5, v6, v7, v8, v9 and v10 exons independently (Fig. 2A). The reverse primer formed a base pair with a constant exon (c7). As shown in Fig. 2B, RT-PCR with 10 primer sets showed that MCF10A and MCF7 cells expressed similar CD44 isoforms. However the expression of CD44v6 isoforms in the MDA-MB-231 cells was different from that in the other two cell lines. Significantly, 
A

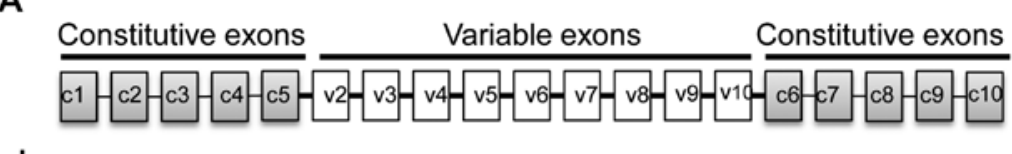

商 c5v6-c7:

B

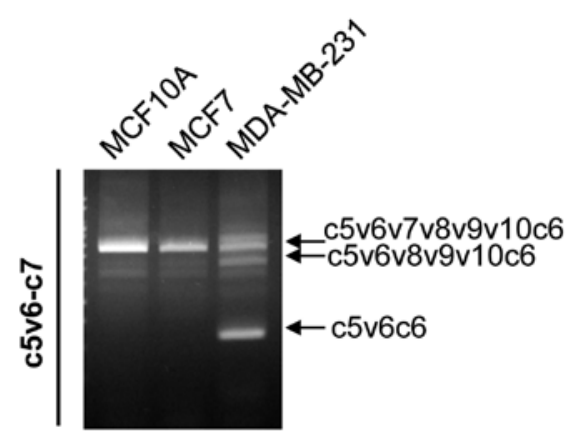

Figure 3. MDA-MB-231 cells show differential expression of CD44v6 isoforms when compared with that of the MCF7 or MCF10A cells. (A) Primer positions are shown. (B) RT-PCR results of CD44v6 using primers shown in A in the MCF10A, MCF7 and MDA-MB-231 cells.

A
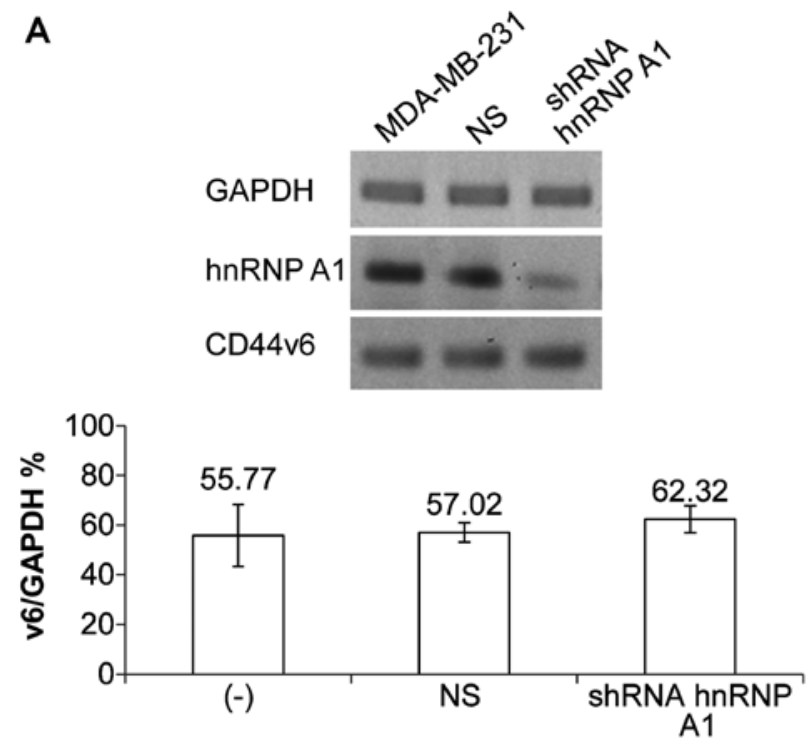

B

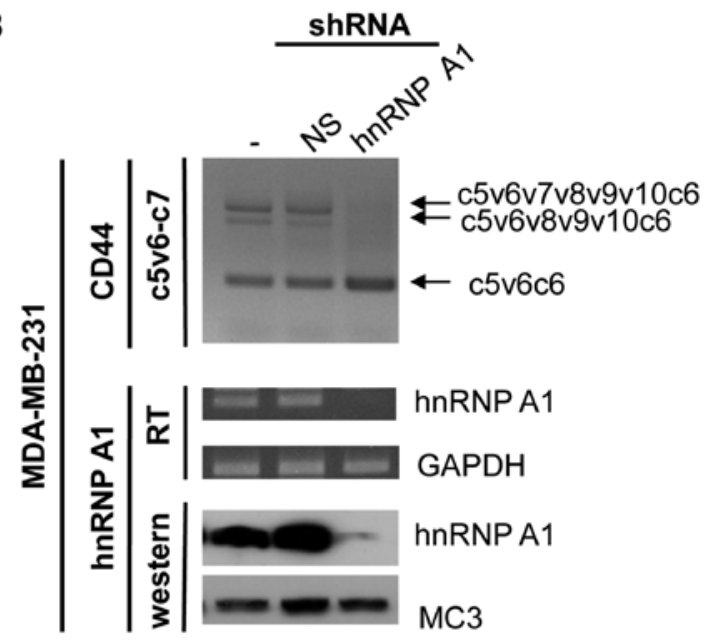

Figure 4. Knockdown of hnRNP A1 reduces the expression of the c5v6v7v8v9v10c6 and c5v6v8v9v10c6 isoforms and increases the expression of the c5v6c6 isoform in MDA-MB-231 cells. (A) Knockdown of hnRNP A1 did not cause an alteration in the expression of CD44v6 mRNA. Quantitative results are shown in the lower panel. (B) Knockdown of hnRNP A1 reduces the expression of the c5v6v7v8v9v10c6 and c5v6v8v9v10c6 isoforms and increases the expression of the c5v6c6 isoform in MDA-MB-231 cells. Reduced expression of hnRNP A1 was determined with RT-PCR and immunoblotting, with GAPDH and MC3 as controls. hnRNP, heterogeneous nuclear ribonucleoprotein.

various low-molecular-weight isoforms were expressed in the RT-PCR analysis with primers $\mathrm{v} 2, \mathrm{v} 3, \mathrm{v} 4, \mathrm{v} 5$ and $\mathrm{v} 6$ (lanes 1,2,3,4 and 5). We decided to focus on CD44v6 in this study. To identify CD44v6 isoforms, we performed RT-PCR analysis using a forward primer that base pair with both the v6 and c5 exon, and a reverse primer that base pair with the c7 exon (Fig. 3A). RT-PCR results showed that MCF10A and MCF7 cells predominantly expressed the c5v6v7v8v9v10c6 isoform (Fig. 3B). However, the MDA-MB-231 cells expressed c5v6v8v9v10c6 and c5v6c6 isoforms, in addition to the $\mathrm{c} 5 \mathrm{v} 6 \mathrm{v} 7 \mathrm{v} 8 \mathrm{v} 9 \mathrm{v} 10 \mathrm{c} 6$ isoform. Therefore we concluded that c5v6v8v9v10c6 and c5v6c6 isoforms are unique to metastatic breast cancer cells (MDA-MB-231).

Knockdown of hnRNP Al reduces the expression of the $c 5 v 6 v 7 v 8 v 9 v 10 c 6$ and $c 5 v 6 v 8 v 9 v 10 c 6$ isoforms and increases the expression of the c5v6c6 isoform in MDA-MB-231 cells. We next aimed to ascertain whether hnRNP A1 knockdown affects the expression of CD44v6 isoforms. Using hnRNP A1-targeting shRNA, we knocked down hnRNP A1 
A

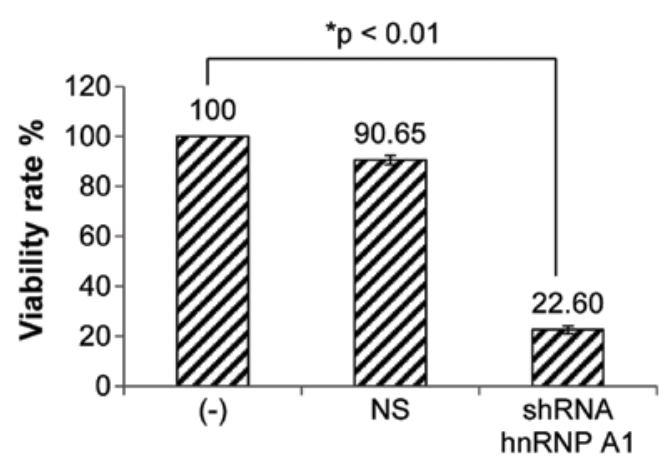

B
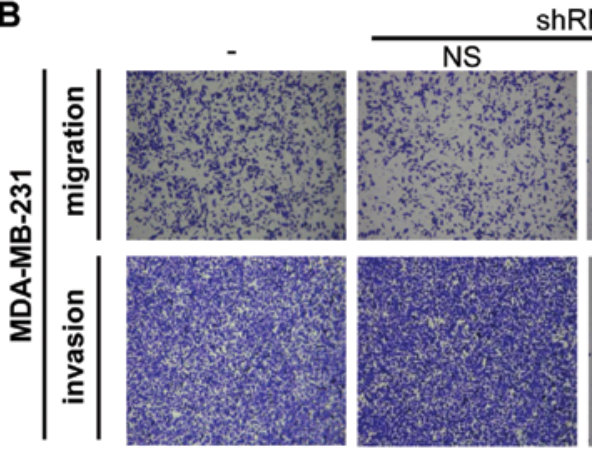

C

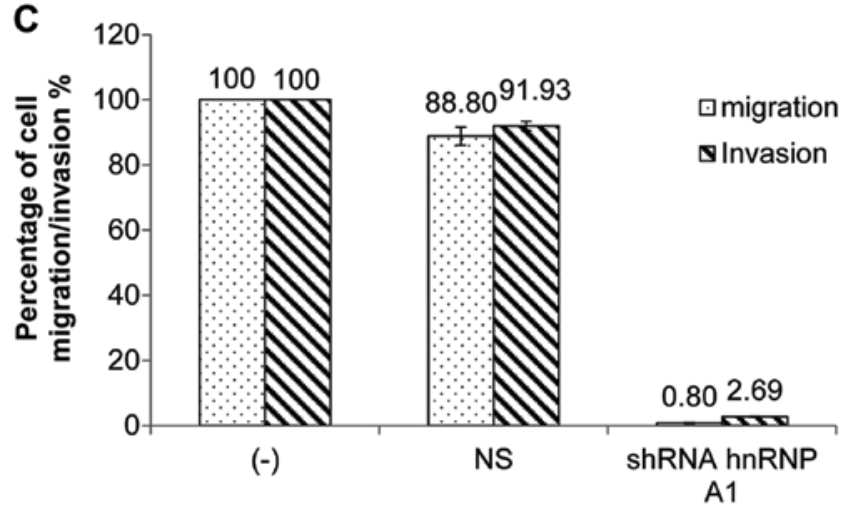

Figure 5. Knockdown of hnRNP A1 reduces the viability and motility of MDA-MB-231 cells. (A) MTT assay is shown following treatment with the hnRNP A1 shRNA virus. (B) Migration and invasion assays are shown (C) Quantitation of the combined results of A and B. hnRNP, heterogeneous nuclear ribonucleoprotein.

expression. hnRNP A1 expression was significantly reduced after the treatment of MDA-MB-231 cells with the shRNA virus of hnRNP A1 as shown with RT-PCR and immunoblot analysis (Fig. 4A, upper panel). By contrast, non-silencing (NS) shRNA did not cause any alteration in hnRNP A1 expression. Fig. 4B shows that reduced expression of hnRNP A1 by shRNA induced a significant decrease in the c5v6v7v8v9v10c6 and c5v6v8v9v10c6 isoforms in the MDA-MB-231 cells. However, expression of the c5v6c6 isoform was significantly increased after treatment with the hnRNP A1 shRNA virus. Infection with non-silencing shRNA did not cause an alteration in CD44v6 expression. Therefore, we concluded that reduced expression of hnRNP A1 has differential effects on the various CD44v6 isoforms.

Knockdown of hnRNP Al reduces cell viability. We next tested the effects of hnRNP A1 knockdown on the cell viability of
MDA-MB-231 cells. The MTT assay results (Fig. 5A) showed that hnRNP A1 knockdown induced a significant decrease in cell viability $(\sim 77.4 \%)$. In contrast to the hnRNP A1 shRNA treatment, non-silencing shRNA did not cause a significant decrease in cell viability.

Knockdown of hnRNP Al reduces the motility of the $M D A-M B-231$ cells. We further tested the effects of hnRNP A1 knockdown on breast cancer cell invasion. We performed invasion and migration assays in the hnRNP A1 shRNA-treated, non-silencing shRNA-treated and non-treated MDA-MB-231 cells. Fig. 5B shows that the hnRNP A1 shRNA-treated cells showed a significant decrease in invasion $(\sim 97.31 \%)$ and migration $(\sim 99.2 \%)$ abilities. By contrast, non-silencing shRNA treatment did not induce significant change both in invasion and migration. A decrease in migration or invasion may have occurred for two reasons. First, hnRNP A1 knockdown may have caused significant cell death. Second, hnRNP A1 knockdown may have induced a decrease in invasion. To confirm the specific reasons, we carefully compared the quantitative results of cell viability and cell invasion. Fig. 5A shows that the decrease in cell invasion ability occurred partially by the decrease in cell viability. Based on the results of Fig. $5 \mathrm{~A}$ and $\mathrm{B}$, we conclude that knockdown of hnRNP A1 induced a decrease in migration ( $21.89 \%)$ and invasion $(\sim 19.9 \%)$.

\section{Discussion}

Alternative splicing of CD44 pre-mRNA produces various combinations of variant exons, thus various proteins with different functions are encoded by a single gene. Among the variants, CD44v6 has attracted attention since transfection of a v6-containing isoform (v4-v7) into a non-metastatic rat pancreatic carcinoma cell line conferred metastatic potential to these cells when infected into syngeneic rats (23). The v6 exon has been shown to promote c-Met activation (21). Treatment of CD44v6 peptides was found to completely abrogate metastatic spread to the lymph nodes and to the lung (31). The function of CD44 isoforms is different in breast cancer cells from pancreatic cells. A study showed that anti-CD44s inhibited breast cancer cell adhesion, motility and invasion while anti-CD44-v6 inhibited cell motility (33). Furthermore, the function of CD44-v6 and the regulatory mechanisms of alternative splicing are not well understood in breast cancer cells. Here, we found that the splicing pattern of the CD44v6 variant in metastatic breast cancer cells (MDA-MB-231) was different from that of non-metastatic breast cancer (MCF7) and normal breast cells (MCF10A). Metastatic MDA-MB-231 cells expressed the c5v6c6 and c5v6v8v9v10c6 isoforms, whereas the MCF7 and MCF10A did not. The c5v6v7v8v9v10c6 isoform was expressed in the MCF10A, MCF7 and MDA-MB-231 cells. Knockdown of hnRNP A1 induced a significant decrease in the $\mathrm{c} 5 \mathrm{v} 6 \mathrm{v} 7 \mathrm{v} 8 \mathrm{v} 9 \mathrm{v} 10 \mathrm{c} 6$ and c5v6v8v9v10c6 isoforms, and an increase in the c5v6c6 isoform. We further found that knockdown of hnRNP A1 induced cell death and significantly inhibited invasion in the MDA-MB-231 cells.

High expression level of CD44-v6 is not related to high metastatic potential of breast cancer cells. Our results showed 
that non-metastatic MCF7 cells expressed the highest level of CD44-v6 isoforms, and MCF10A and MDA-MB-231 cells expressed a lower level of CD44-v6. Our results indicate that the expression level of CD44-v6 is not linked to the metastatic potential in human breast cancer cells.

Expression of the c5v6c6 isoform is unique to $M D A-M B$ 231 cells. Our RT-PCR results with various primer sets indicate that expression of the CD44 isoforms is similar in MCF10A and MCF7 cells. However, we found that CD44 expression in the MDA-MB-231 cells was different than that in the MCF10A and MCF7 cells. In this study, we carefully observed expression of the CD44v6 isoform in these three cell lines. Our results indicate that the MCF10A and MCF7 cells expressed only the c5v6v7v8v9v10c6 isoform at a significant level. However, the MDA-MB-231 cells, in addition to expressing the c5v6v7v8v9v10c6 isoform, also expressed the c5v6c6 and c5v6v8v9v10c6 isoforms. Based on the fact that MDA-MB-231 is a cell line with high metastatic potential, and that c5v6v8v9v10c6 and c5v6c6 isoforms are only expressed in MDA-MB-231 cells, we hypothesized that the c5v6v8v9v10c6 and c5v6c6 isoforms of CD44 confer metastatic potential.

Effects of hnRNP A1 knockdown on CD44v6 expression is different in breast cancer cells and hepatocellular carcinomas. We found that hnRNP A1 knockdown did not induce a significant change in the total CD44v6 expression level. This result is contrary to the effects of hnRNP A1 knockdown on CD44v6 expression in hepatocellular carcinomas (31). In hepatocellular carcinoma, patients with high hnRNP A1 tended to have higher levels of CD44v6. Our results indicated that the regulation of CD44 splicing is different in breast cancer cells from hepatocellular carcinomas. hnRNP A1 knockdown induces the switching of CD44v6 isoforms to others, without reducing the expression level of the CD44v6 isoforms. We found that hnRNP A1 knockdown induced cell death at a significant level. We showed that hnRNP A1 knockdown also induced a decrease in invasive ability. The effects of hnRNP A1 knockdown on the viability of hepatocellular carcinoma cells were not tested in the previous study (31). Thus, the relative effect of hnRNP A1 knockdown on invasion is not clearly shown. We quantified the effects of hnRNP A1 knockdown on cell viability and invasion systematically. We conclude that hnRNP A1 knockdown reduced both cell viability and invasion. Thus, the effect of hnRNP A1 knockdown on CD44v6 expression is different in breast cancer cells than that in hepatocellular carcinomas.

Reduced expression of hnRNP Al reduces expression of the c5v6v7v8v9v10c6 and c5v6v8v9v10c6 isoforms and significantly promotes the c5v6c6 isoform in MDA-MB-231 cells. Knockdown of hnRNP A1 differentially regulates CD44v6 splicing in breast cancer cells. We found that, after knockdown of hnRNP A1 in the MDA-MB-231 cells, expression of the c5v6v7v8v9v10c6 and c5v6v8v9v10c6 isoforms was significantly decreased. In contrast, expression of the c5v6c6 isoform was significantly increased after hnRNP A1 knockdown. The functions of these CD44 splicing isoforms warrant confirmation.

\section{Acknowledgements}

This study was supported by the NRF-2013-R1A1A2062582 grant (to H.S.) and the NRF-2013-R1A1A2061321 grant (to X.Z.) funded by the National Research Foundation (NRF) of the Korean Ministry of Education, Science, and Technology (MEST) and the Integrative Aging Research Center of Gwangju Institute of Science and Technology (GIST).

\section{References}

1. Modrek B and Lee C: A genomic view of alternative splicing. Nat Genet 30: 13-19, 2002.

2. Moon H, Cho S, Yang X, Zhou J, Loh TJ, Zheng X and Shen H: Identification of novel splicing variants from RON proto-oncogene pre-mRNA. Oncol Rep 28: 2217-2220, 2012.

3. Shen H, Zheng X, Luecke S and Green MR: The U2AF35-related protein Urp contacts the 3 ' splice site to promote U12-type intron splicing and the second step of U2-type intron splicing. Genes Dev 24: 2389-2394, 2010 .

4. Shen $\mathrm{H}$ and Green MR: RS domain-splicing signal interactions in splicing of U12-type and U2-type introns. Nat Struct Mol Biol 14: 597-603, 2007.

5. Lee J, Zhou J, Zheng X, Cho S, Moon H, Loh TJ, Jo K and Shen H: Identification of a novel cis-element that regulates alternative splicing of Bcl-x pre-mRNA. Biochem Biophys Res Commun 420: 467-472, 2012.

6. Cho S, Moon H, Yang X, Zhou J, Kim HR, Shin MG, Loh TJ, Zheng $X$ and Shen H: Validation of trans-acting elements that promote exon 7 skipping of SMN2 in SMN2-GFP stable cell line. Biochem Biophys Res Commun 423: 531-535, 2012.

7. Chen M and Manley JL: Mechanisms of alternative splicing regulation: Insights from molecular and genomics approaches. Nat Rev Mol Cell Biol 10: 741-754, 2009.

8. Matlin AJ, Clark F and Smith CW: Understanding alternative splicing: Towards a cellular code. Nat Rev Mol Cell Biol 6: 386-398, 2005.

9. Cáceres JF and Kornblihtt AR: Alternative splicing: Multiple control mechanisms and involvement in human disease. Trends Genet 18: 186-193, 2002.

10. Faustino NA and Cooper TA: Pre-mRNA splicing and human disease. Genes Dev 17: 419-437, 2003

11. Cooper TA, Wan L and Dreyfuss G: RNA and disease. Cell 136: 777-793, 2009.

12. Kansas GS, Wood GS and Dailey MO: A family of cell-surface glycoproteins defined by a putative anti-endothelial cell receptor antibody in man. J Immunol 142: 3050-3057, 1989.

13. Pals ST, Hogervorst F, Keizer GD, Thepen T, Horst E and Figdor CC: Identification of a widely distributed $90-\mathrm{kDa}$ glycoprotein that is homologous to the Hermes-1 human lymphocyte homing receptor. J Immunol 143: 851-857, 1989.

14. Picker LJ, Nakache M and Butcher EC: Monoclonal antibodies to human lymphocyte homing receptors define a novel class of adhesion molecules on diverse cell types. J Cell Biol 109: 927-937, 1989.

15. Fox SB, Fawcett J, Jackson DG, Collins I, Gatter KC, Harris AL, Gearing A and Simmons DL: Normal human tissues, in addition to some tumors, express multiple different CD44 isoforms. Cancer Res 54: 4539-4546, 1994

16. Screaton GR, Bell MV, Bell JI and Jackson DG: The identification of a new alternative exon with highly restricted tissue expression in transcripts encoding the mouse Pgp-1 (CD44) homing receptor. Comparison of all 10 variable exons between mouse, human, and rat. J Biol Chem 268: 12235-12238, 1993.

17. Ponta H, Wainwright D and Herrlich P: The CD44 protein family. Int J Biochem Cell Biol 30: 299-305, 1998.

18. Hofmann M, Rudy W, Günthert U, Zimmer SG, Zawadzki V, Zöller M, Lichtner RB, Herrlich P and Ponta H: A link between ras and metastatic behavior of tumor cells: Ras induces CD44 promoter activity and leads to low-level expression of metastasis-specific variants of CD44 in CREF cells. Cancer Res 53: 1516-1521, 1993.

19. Weg-Remers S, Ponta H, Herrlich P and König H: Regulation of alternative pre-mRNA splicing by the ERK MAP-kinase pathway. EMBO J 20: 4194-4203, 2001. 
20. Matzke A, Herrlich P, Ponta $\mathrm{H}$ and Orian-Rousseau V: A five-amino-acid peptide blocks Met- and Ron-dependent cell migration. Cancer Res 65: 6105-6110, 2005.

21. Orian-Rousseau V, Chen L, Sleeman JP, Herrlich P and Ponta H: CD44 is required for two consecutive steps in HGF/c-Met signaling. Genes Dev 16: 3074-3086, 2002.

22. Tremmel M, Matzke A, Albrecht I, Laib AM, Olaku V, Ballmer-Hofer K, Christofori G, Héroult M, Augustin HG, Ponta $\mathrm{H}$, et al: A CD44v6 peptide reveals a role of CD44 in VEGFR-2 signaling and angiogenesis. Blood 114: 5236-5244, 2009.

23. Günthert U, Hofmann M, Rudy W, Reber S, Zöller M, Haussmann I, Matzku S, Wenzel A, Ponta $\mathrm{H}$ and Herrlich P: A new variant of glycoprotein CD44 confers metastatic potential to rat carcinoma cells. Cell 65: 13-24, 1991.

24. Heider KH, Kuthan H, Stehle G and Munzert G: CD44v6: A target for antibody-based cancer therapy. Cancer Immunol Immunother 53: 567-579, 2004.

25. Louderbough JM and Schroeder JA: Understanding the dual nature of CD44 in breast cancer progression. Mol Cancer Res 9: 1573-1586, 2011.

26. Hill A, McFarlane S, Mulligan K, Gillespie H, Draffin JE, Trimble A, Ouhtit A, Johnston PG, Harkin DP, McCormick D, et al: Cortactin underpins CD44-promoted invasion and adhesion of breast cancer cells to bone marrow endothelial cells. Oncogene 25: 6079-6091, 2006.
27. Ouhtit A, Abd Elmageed ZY, Abdraboh ME, Lioe TF and Raj MH: In vivo evidence for the role of CD44s in promoting breast cancer metastasis to the liver. Am J Pathol 171: 2033-2039, 2007.

28. Busch A and Hertel KJ: Evolution of SR protein and hnRNP splicing regulatory factors. Wiley Interdiscip Rev RNA 3: 1-12, 2012.

29. Mayeda A, Munroe SH, Cáceres JF and Krainer AR: Function of conserved domains of hnRNP A1 and other hnRNP A/B proteins. EMBO J 13: 5483-5495, 1994.

30. Martinez-Contreras R, Cloutier P, Shkreta L, Fisette JF, Revil T and Chabot B: hnRNP proteins and splicing control. Adv Exp Med Biol 623: 123-147, 2007.

31. Zhou ZJ, Dai Z, Zhou SL, Fu XT, Zhao YM, Shi YH, Zhou J and Fan J: Overexpression of HnRNP A1 promotes tumor invasion through regulating CD44v6 and indicates poor prognosis for hepatocellular carcinoma. Int J Cancer 132: 1080-1089, 2013.

32. van Weering DH, Baas PD and Bos JL: A PCR-based method for the analysis of human CD44 splice products. PCR Methods Appl 3: 100-106, 1993.

33. Afify A, Purnell P and Nguyen L: Role of CD44s and CD44v6 on human breast cancer cell adhesion, migration, and invasion. Exp Mol Pathol 86: 95-100, 2009. 